01,02

\title{
Влияние распределения волновой функции на диссоциацию экситонов в электрическом поле
}

\author{
(C) J. Heckötter ${ }^{1}$, M. Freitag ${ }^{1}$, D. Fröhlich ${ }^{1}$, M. Aßmann ${ }^{1}$, M. Bayer ${ }^{1,2}$, M.A. Семина' ${ }^{2}$, М.М. Глазов \\ ${ }^{1}$ Experimentelle Physik 2, Technische Universität Dortmund, \\ Dortmund, Germany \\ ${ }^{2}$ Физико-технический институт им. А.Ф. Иофффе РАН, \\ Санкт-Петербург, Россия \\ ฯ E-mail: glazov@coherent.ioffe.ru
}

\begin{abstract}
Показано, что порог диссоциации экситона - связанной электрон-дырочной пары - в электрическом полем определяется, главным образом, его энергией: как и ожидалось, напряжение диссоциации уменьшается с ростом энергии экситона. Однако в мультиплетах состояний, принадлежащих определенному главному квантовому числу $n$, напряжение диссоциации возрастает с ростом энергии состояния, что противоречит ожиданиям, основанным на энергетических соображениях. Такое поведение продемонстрировано на желтой экситонной серии в $\mathrm{Cu}_{2} \mathrm{O}$, оно связано с перераспределением волновой функции в потенциальном рельефе, где верхнее (нижнее) по энергии состояние сдвигается к (от) туннельному барьеру.
\end{abstract}

Мы благодарим Немецкое научное общество за поддержку в рамках программы ICRC TRR 160 (проект A1) и проекта № AS 459/1-3. Мы признательны за частичную поддержку РФФИ (15-52-12012), гранту Президента РФ МД-1555.2017.2 и программе Президиума РАН „Наноструктуры: физика, химия, биология, основы технологии“. М.В. также благодарен за поддержку гранту Правительства РФ 14.Z50.31.0021.

DOI: $10.21883 /$ FTT.2018.08.46235.10Gr

\section{1. Введение}

В последнее время детально изучено поведение желтой экситонной серии в закиси меди $[1,2]$ в электрическом поле [3-5], были выявлены сильная анизотропия электрооптических свойств этого типичного кубического кристалла, нетривиальная тонкая структура экситонных состояний, перераспределение силы осциллятора между состояниями под действием поля, а также скейлинговые закономерности в зависимости от главного квантового числа для разных величин [6]. В этой же работе наблюдалась диссоциация экситонов в достаточном большом электрическом поле. В общих чертах проявление диссоциации экситонов было ожидаемым: пороговое напряжение диссоциации понижается с ростом главного квантового числа состояния. Такое поведение согласуется с качественными аргументами, свидетельствующими о том, что чем выше абсолютная энергия состояния (т.е., чем больше главное квантовое число), тем данное состояние более подвержено воздействию электрического поля. Однако, более подробно диссоциация не исследовалась. Исследование поведения экситонов во внешних полях по сравнению с атомами облегчено изза меньшей ридберговской энергии экситонов. Поэтому влияние внешних полей наблюдается при значительно меньшей их напряженности и по той же причине систематическое описание эффектов может быть легко получено для всей серии экситонных состояний.

Здесь мы более подробно исследуем диссоциацию экситонов в однородном электрическом поле, которое приводит к наклону кулоновского потенциала, описывающего связанные состояния экситона. Внутри набора уровней Штарка, связанных с данным главным квантовым числом $n$, напряжение, требуемое для диссоциации экситона, возрастает с номером состояния, это противоречит анализу, основанному на значениях энергий связи состояний. Объяснение этого наблюдения требует более подробного анализа, который показывает, что экспериментальные данные можно понять, на основе распределения волновых функций в потенциальном рельефе.

\section{2. Эксперимент}

Эксперименты проводились на кристалле закиси меди, ориентированном вдоль направления [110]. Он был помещен в держатель, обеспечивающий отсутствие упругих напряжений, с помощью которого можно было прикладывать электрическое поле при температуре $T=1.3 \mathrm{~K}[7,8]$. Поле было приложено в продольной конфигурации, т. е. вдоль оптической оси, совпадающей с направлением [110]. Поглощение измерялось с помощью широкополосного источника белого света. Свет был линейно поляризован вдоль направления [001], так что помимо дипольно-разрешенных $P$-экситонов можно было наблюдать квадрупольно-разрешенные переходы, связанные с $S$ - и $D$-экситонами [5]. После прохождения через кристалл белый свет диспергировался двойным монохроматором и детектировался кремниевой камерой с зарядовой связью, при этом обеспечивалось спектральное разрешение менее $10 \mu \mathrm{eV}$.

На рис. 1, $a$, верхняя панель, показана наблюдаемая в эксперименте эволюция экситонных мультиплетов, связанных с $n=5$ и 6 в электрическом поле. Здесь и далее мы сохраняем обозначения водородоподобной 


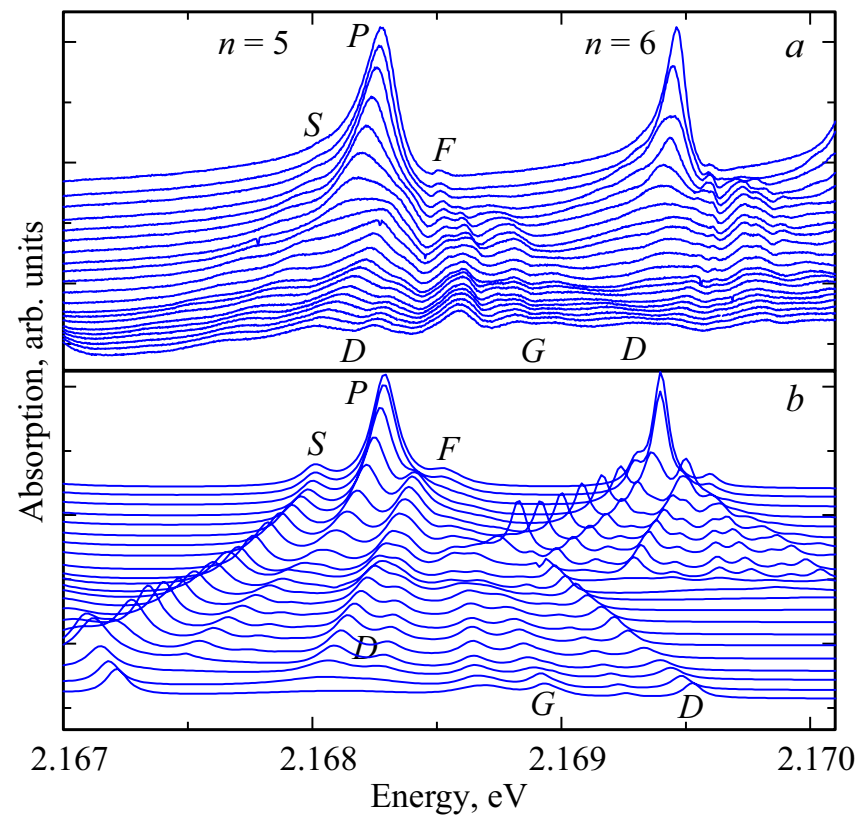

Рис. 1. (a) спектр поглощения в дипазоне энергий экситонов с $n=5$ и 6 при различных напряжениях, возрастающих от $U=0$ до $20 \mathrm{~V}$ с шагом в $1 \mathrm{~V}$, приложенных вдоль направления [110]. Свет был линейно поляризован по оси [001]. (b) рассчитанный спектр поглощения для состояний с $n=5$ и 6 , подробности приведены в [15].

серии $S, P, D, \ldots$ для экситонных состояний с орбитальным моментом $l=0,1,2, \ldots$, хотя эти состояния смешиваются даже в нулевом поле из-за кристаллического окружения $[9,10]$, а также дополнительно смешиваются в электрическом поле. В спектре при нулевом поле главными являются $P$-экситоны с $n=5$ и $n=6$, каждый из которых сопровождается слабой высокоэнергетической особенностью, связанной с $F$-экситонами $[9,10]$. При приложении поля поведение мультиплетов схожее, поэтому мы в основном фокусируемся на случае $n=5$, поскольку для него легче разрешить изменения. С ростом поля $P$-экситон демонстрирует квадратичный штарковский сдвиг в сторону меньших энергий, а $F$-экситон слабо смещается в сторону более высоких энергий. Также в спектре появляются дополнительные особенности из-за смешивания состояний, обусловленного понижением симметрии, индуцированным полем. Например, на низкоэнергетической стороне $P$-экситона имеется слабое плечо, связанное с квадрупольно-разрешенным $S$-экситоном, которое становится более выраженным в ненулевом поле. Оно сдвигается в основном параллельно $P$-экситону. В сильных полях оба экситона пропадают, причем $S$-экситон исчезает при меньшей напряженности поля, чем $P$-экситон.

Между $P$ - и $F$-экситонами появляется дублет $D$-состояний, сила осциллятора которого достигает максимума в промежуточных полях, а затем уменьшается с ростом поля. Удивительно, что эти две особенности, расположенные выше по энергии $S$ - и $P$-экситонов, сохраняются даже в том диапазоне полей, где $S$ и $P$-экситоны практически исчезли. При более высоких энергиях в широких энергетических полосах, составленных из множества отдельных линий, разрешаются $F$ - и $G$-экситоны. Заметим, что существует также $D$-экситон, расположенный над этими состояниями, который сильно сдвигается в сторону бо́льших энергий с увеличением напряжения. Подтверждая только что описанный удивительный результат, эти линии с высокой энергией имеют тенденцию к увеличению интенсивности вплоть до максимального приложенного напряжения.

Как уже упоминалось, для мультиплета $n=6$ мы наблюдаем аналогичную эволюцию спектров в электрическом поле. Однако, для достижения сопоставимых расщеплений требуется приложение меньших напряжений, причем особенности при больших энергиях более стабильны, чем особенности на низкоэнергетической стороне. Кроме того, диссоциация происходит в меньших полях, чем те, которые требуются для экситонов с $n=5$, поэтому мультиплет с $n=6$ полностью исчез при тех напряжениях, когда мультиплет с $n=5$ еще заметен.

Подводя короткий промежуточный вывод на основе этих экспериментальных данных, можно заключить, что диссоциация в пределах мультиплета с данным $n$ демонстрирует противоположную тенденцию с ростом энергии по сравнению состояниями из разных мультиплетов.

\section{3. Обсуждение}

Для того, чтобы количественно описать этот результат, мы рассмотрим водородоподобную модель, пренебрегая особенностями зонной структуры полупроводника [9-13]; в качестве подробного обзора см. [14]. Этот приближенный подход позволяет сделать качественные и полуколичественные выводы о диссоциации экситонов. Водородоподобный гамильтониан относительного движения электрона и дырки в электрическом поле $\mathbf{E}$, приложенном вдоль оси $z$, имеет вид

$$
\mathscr{H}_{0}=\frac{\mathbf{p}^{2}}{2 \mu}-\frac{e^{2}}{\varepsilon r}-e E z,
$$

и описывает относительное движение отрицательно заряженной частицы в потенциале, состоящем из кулоновского потенциала и потенциала электрического дипольного взаимодействия с полем $\mathbf{E}=(0,0, E)$. Здесь $\mathbf{r}=(x, y, z)-$ относительная координата электрона и дырки, $\mathbf{p}=-\mathrm{i} \hbar \partial / \partial \mathbf{r}-$ импульс относительного движения, $\mu$ - приведенная масса электрона и дырки, и $\varepsilon-$ диэлектрическая проницаемость оксида меди.

Для простоты пренебрегаем любым расщеплением экситонных состояний в нулевом поле [10,12], так что можно применять вырожденную теорию возмущений. В слабых электрических полях собственные состояния являются линейными комбинациями состояний в нулевом поле. Из-за этого смешивания состояния приобретают электрический дипольный момент, который 


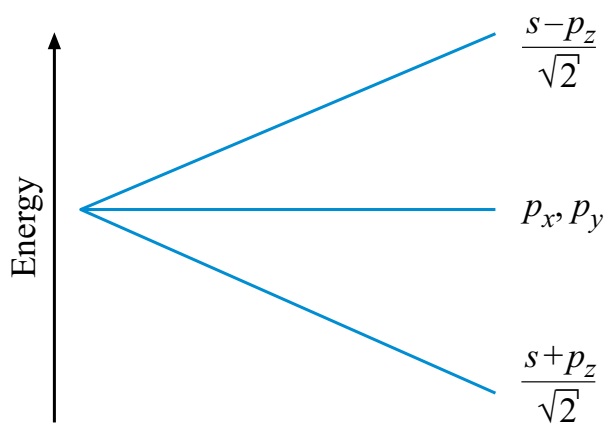

$a$
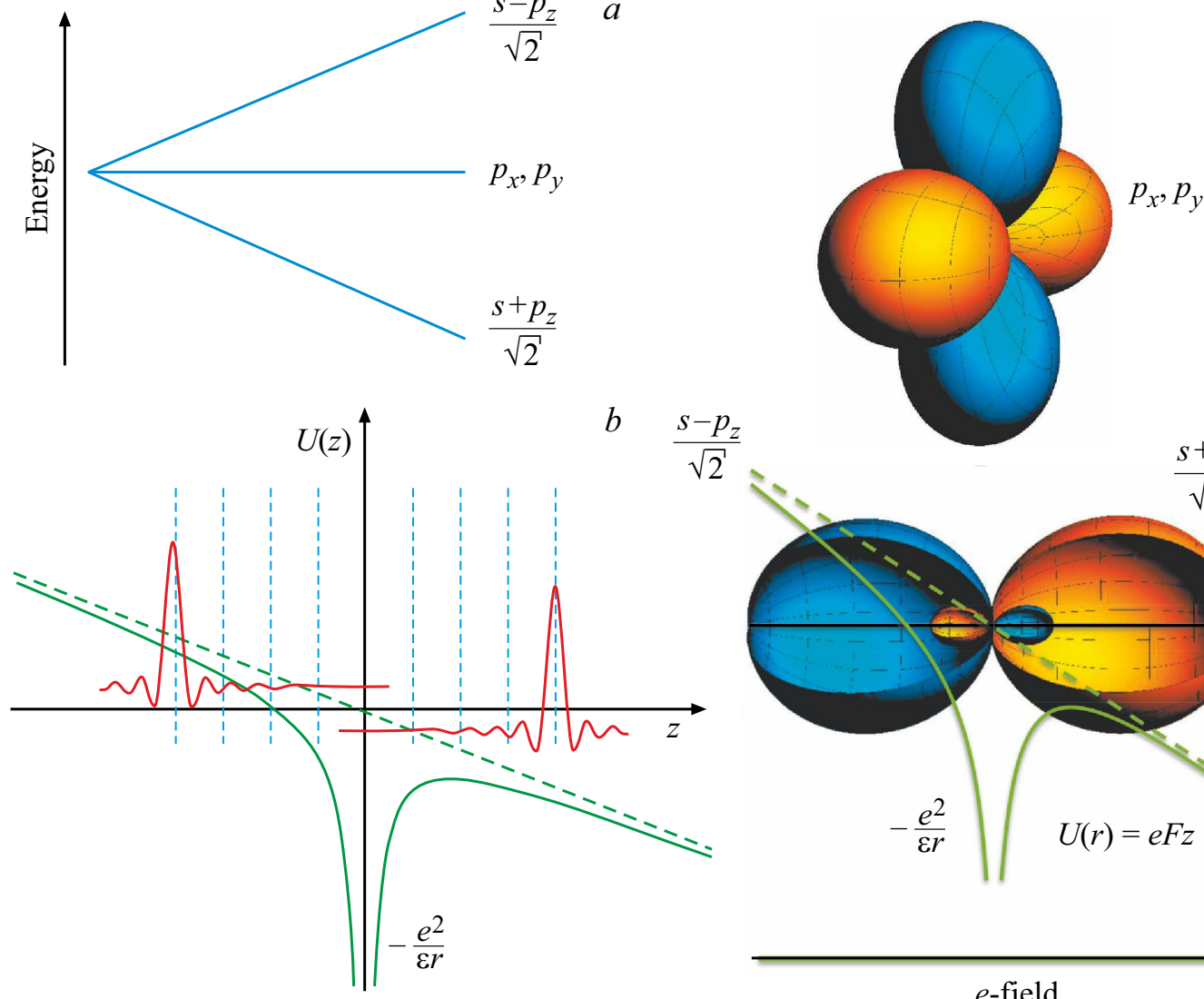

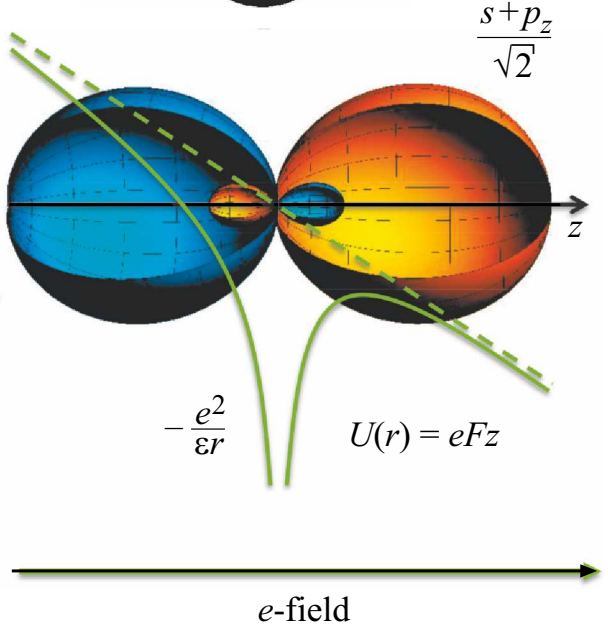

Рис. 2. $(a)$ - иллюстрация штарковских состояний, т. е. зависимости энергии экситона от напряженности электрического поля для мультиплета с $n=2$. Справа в виде линейных комбинаций водородоподобных состояний нулевого поля показаны состояния в конечном поле. $(b)$ - схематическое представление волновых функций в электрическом поле с учетом наклона кулоновского потенциала под действием внешнего поля (сплошная линия) и с учетом только электрического дипольного взаимодействия (штриховая линия). (c) - изображения волновых функций в электрическом поле для симметричного (антисимметричного) состояния с меньшей (большей) энергией, демонстрирующее облегченное туннелирование нижнего состояния по сравнению с верхним.

ориентируется под действием электрического поля, что приводит к линейному сдвигу энергии с увеличением напряженности поля (без учета взаимодействия между состояниями с различными $n$ ), как схематически показано на рис. 2, $a$ для $n=2$. Мы выбрали это состояние для иллюстрации из-за небольшого числа уровней в мультиплете, а именно четное $S$-состояние $(l=0)$ и три нечетных $P$-состояния с $l=1$. Каждое отдельное состояние имеет сферически-симметричное распределение заряда.

В электрическом поле состояния противоположной четности смешиваются. В случае $n=2$ итоговые суперпозиции состояний имеют довольно простой вид. $P$-орбитали, $P_{x}$ и $P_{y}$, не смешиваются с другими состояниями, так как они ориентированы по нормали к полю, тем самым они не испытывают энергетического сдвига из-за отсутствующего дипольного взаимодействия. Напротив, $S$ - и $P_{z}$-состояния перемешиваются, за счет чего симметричная (антисимметричная) комбинация смещается в сторону низких (высоких) энергий, в соответствии с изложенными выше соображениями. В зависимости от относительного знака суперпозиция приводит к увеличению (уменьшению) волновой функции с одной стороны от плоскости $z=0$, в то время как на другой стороне поведение обратное: амплитуда волновой функции уменьшается (увеличивается). Это приводит к асимметрии распределения заряда двух состояний вдоль $z$, причем дипольный момент состояния направлен из области с большй электронной плотностью в область с меньшей плотностью, см. рис. $2, b$.

Эти перераспределения волновых функций должны быть связаны с потенциальным рельефом в электрическом поле на рис. 2, $b$. Полный потенциал понижается для положительных $z$, поэтому формируется туннельный барьер. Вероятность диссоциации определяется туннельной экспонентой, которая связана с квазиклассическим подбарьерным действием $\mathcal{S}=\int p_{z} d z$ под барьером. Для симметричного состояния с дипольным моментом, ориентированным вдоль электрического поля, энергия понижается. Это состояние с повышенной вероятностью обнаружения заряда на правой стороне центробежного барьера, т.е., при положительных $z$, поэтому такое состояние может диссоциировать при сравнительно более 


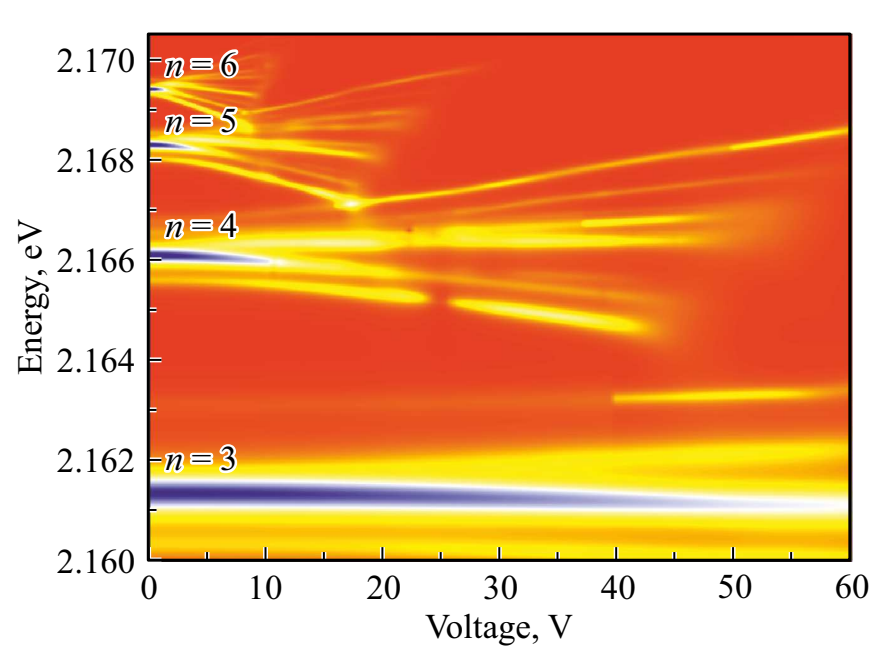

Рис. 3. Контурный график с рассчитанным спектром поглощения в зависимости от напряжения, приложенного по оси [110]. Возбуждающий свет поляризован по оси [001]. Подробности см. в $[15]$.

низких напряжениях, несмотря на его пониженную энергию. Напротив, для антисимметричного состояния, для которого распределение вероятности противоположное по сравнению с симметричным состоянием, туннелирование оказывается менее вероятным, несмотря на его большую энергию. Это объясняет парадоксальное наблюдение того, что для диссоциации экситонов с более высокими энергиями требуются большие электрические поля.

Этот качественный анализ подтверждается теоретическими расчетами, подробности которых приведены в другой работе [15]. Нижняя панель рис. 1 показывает рассчитанный для сопоставления с экспериментом спектр поглощения мультиплетов с $n=5$ и $n=6$. Из-за немного меньшей ширины линии, заложенной в расчете, такие особенности как $S$-состояния на низкоэнергетическом крыле в теоретических графиках легче различить, чем в экспериментальных. Расчеты достаточно хорошо воспроизводят эволюцию линий, в частности, в отношении поведения напряжения диссоциации для различных состояний.

Рис. 3 показывает в виде контурного графика рассчитанные в рамках модели [15] спектры поглощения в энергетическом диапазоне экситонных состояний с $n=3,4,5$ и 6 в зависимости от приложенного напряжения. Моделирование показывает расщепление различных водородоподобных состояний в серию штарковских уровней в электрическом поле с увеличением числа уровней с ростом главного квантового числа. Расчеты также подтверждают описанное выше уменьшение напряжения диссоциации при увеличении $n$, т.е. энергии экситонов, примерно в 2 раза при переходе от данного мультиплета к соседнему. В то же время, в пределах мультиплета с заданным $n$ это напряжение увеличивается от с ростом энергии состояния в хорошем согласии с экспериментом.
Таким образом, мы изучили диссоциацию экситонов желтой серии в закиси меди под действием электрического поля. В отличие от простых ожиданий, мы показали, что не только энергия состояния имеет значение для диссоциации в поле, но и распределение волновой функции в суммарном потенциале. Это приводит к противоположным тенденциям в зависимости напряжения диссоциации от энергии для состояний с одинаковыми и различными главными квантовыми числами $n$.

\section{Список литературы}

[1] Е.Ф. Гросс, Н.А. Каррыев. ДАН СССР 84, 471 (1952).

[2] E.F. Gross. Il Nuovo Cimento 4, 672 (1956).

[3] Е.Ф. Гросс, Б.П. Захарченя, Н.М. Рейнов. ДАН СССР 97, 57 (1954).

[4] V.T. Agekyan, B.S. Monozon, I.P. Shiryapov. Phys. Status Solidi B 66, 359 (1974); V.T. Agekyan. Phys. Status Solidi A 43, 11 (1977).

[5] J. Heckötter, M. Freitag, D. Fröhlich, M. Aßmann, M. Bayer, M.A. Semina, M.M. Glazov. Phys. Rev. B 95, 035210 (2017).

[6] J. Heckötter, M. Freitag, D. Fröhlich, M. Aßmann, M. Bayer, M.A. Semina, M.M. Glazov. Phys. Rev. B 96, 125142 (2017).

[7] T. Kazimierczuk, D. Fröhlich, S. Scheel, H. Stolz, M. Bayer. Nature (London) 514, 343 (2014).

[8] J. Brandt, D. Fröhlich, C. Sandfort, M. Bayer, H. Stolz, N. Naka. Phys. Rev. Lett. 99, 217403 (2007).

[9] Ch. Uihlein, D. Fröhlich, R. Kenklies. Phys. Rev. B 23, 2731 (1981).

[10] J. Thewes, J. Heckötter, T. Kazimierczuk, M. Aßmann, D. Fröhlich, M. Bayer, M.A. Semina, M.M. Glazov. Phys. Rev. Lett. 115, 027402 (2015).

[11] F. Schweiner, J. Main, M. Feldmaier, G. Wunner, C. Uihlein. Phys. Rev. B 93, 195203 (2016).

[12] F. Schöne, S.-O. Krüger, P. Grünwald, H. Stolz, S. Scheel, M. Aßmann, J. Heckötter, J. Thewes, D. Fröhlich, M. Bayer. Phys. Rev. B 93, 075203 (2016).

[13] S. Zielińska-Raczyńska, D. Ziemkiewicz, G. Czajkowski. Phys. Rev. B 94, 045205 (2016).

[14] М.А. Семина. ФТТ 60, 7, 1515 (2018).

[15] J. Heckötter, M. Freitag, D. Fröhlich, M. Aßmann, M. Bayer, M.A. Семина, M.M. Глазов. Phys. Rev. B. В печати.

Редактор Ю.Э. Китаев 